\title{
An Optimization Analysis on Testing Method for Cooking Oil Fumes Control Equipment in China
}

\author{
Jun-Xiao $^{1}$, Lihua-Wang ${ }^{2}$, Yan-Liu ${ }^{1}$, Haien-Xiang ${ }^{1}$ \\ ${ }^{1}$ China National Center for Quality Supervision and Test of Environmental Protection Products, Shijiazhuang, China \\ ${ }^{2}$ Hebei Aijie Environmental Protection Technology Co. Ltd., Shijiazhuang, China
}

Email address:

xiaojunhby@163.com (Jun-Xiao)

To cite this article:

Jun-Xiao, Lihua-wang, Yan-Liu, Haien-Xiang. An Optimization Analysis on Testing Method for Cooking Oil Fumes Control Equipment in China. International Journal of Environmental Protection and Policy. Vol. 6, No. 2, 2018, pp. 32-35. doi: 10.11648/j.ijepp.20180602.12

Received: May 15, 2018; Accepted: May 31, 2018; Published: June 19, 2018

\begin{abstract}
Cooking Oil fumes have become one of the most important pollutants in China's society. Oil fume purification equipment has emerged as the times require. China has issued inspection standards for the quality control of such products, but these standards have some drawbacks such as poor operation, low test accuracy, and poor data reproducibility. According to actual work experience, this method is based on the sampling process and the extraction solution. The recommendations for optimization of the air-tightness, air-tightness, exhaust emissions, and testing instruments are provided in 5 areas, which provide references for the quality inspection of such products.
\end{abstract}

Keywords: COFs Control Equipment, Testing Method, Optimization Analysis

\section{Introduction}

With the vigorous development of the tertiary industry, the market has more and more demand for the catering industry, and the pollution caused by soot in the catering industry has become more and more obvious. Many cities complain about more than $40 \%$ of complaints about environmental pollution and become cities. One of the three major public hazards. Cooking Oil Fumes (COFs) refers to the volatile oils, organic matter, and their thermal decomposition or cracking products during food cooking and processing [1]. It is a component and its complex aerosol, in the form of gaseous, liquid and solid tri-state mixed suspensions, including $\mathrm{NO}_{\mathrm{X}}, \mathrm{SO}_{2}$, soot particles, volatile organic compounds (VOCs), waste gas waste liquids, etc., which are greatly harmful to human tissues.

The fume pollution in the catering industry has long attracted the attention of the government. Article 44 of the Air Pollution Prevention and Control Law promulgated at the beginning of the century clearly stipulates that operators of urban catering services must take measures to prevent smoke from polluting the living environment of nearby residents. Therefore, the fume purification equipment has become the primary weapon to control fume pollution, and its demand has increased year by year. The efficient fume purification method is also a research hotspot in the society. However, China's current national standards have some disadvantages such as difficult operation, low test accuracy, and poor data reproducibility in the detection of fume purification equipment product quality. According to actual work experience, the current inspection methods for fume extraction equipment are analyzed. Optimization recommendations to better provide accurate data support for the monitoring and supervision of cooking fume purification.

\section{Status Quo of Oil Fume Purification Equipment in China}

China has a wide variety of foods and cooking methods. Its cooking fumes are complex. Compared with Western foods, the produced fumes are of high concentration and complex in composition, and most of the restaurants in China have low investment costs. Therefore, the fume purification equipment used has a low level of technology. At present, domestic fume purification The following types of equipment:

(1) Mechanical and physical purification equipment. Method of removing soot by filtration, inertial collisions, adsorption or other mechanical separation principles. The principle is simple, low cost, easy to maintain, and used for 
pretreatment of fume purification.

(2) High-voltage electrostatic purification equipment. Application of electrostatic deposition to remove soot. Soot particles enter the equipment and are charged. They are deposited on the polar plates under the force of electric field and gravity. This method is mature and has a wide application range. It is one of the commonly used methods for oil fume purification equipment.

(3) Wet spray cleaning equipment. Application of water film, spray, impact and other liquid absorption principles to remove fumes. Although this method has high processing efficiency and can remove flue gas and most of organic pollutants, the energy consumption in the purification process is high and it is easy to cause secondary pollution in the environment, and it is gradually replaced by other methods.

(4) Compound purification purification equipment. Is the use of two or more purification methods, the combination of the common method of fume purification. Nowadays, most of purification equipment use not only a single purification method, such as the use of high-pressure electrostatic method, but also use physical methods such as filtration or adsorption to enhance the overall purification capability of the equipment. Therefore, this method is used more often in oil fume purification equipment.

The purification efficiency of the fume purification equipment adopting the above purification principle is different, and the level of the purification efficiency cannot be judged according to the adopted method. In order to strengthen the regulation of soot pollution, a scientific and effective detection method is to verify the purification performance and ensure that the fume purification equipment can meet the requirements. The important means of emission standards.

\section{Analysis of Detection Methods of Current Fume Purification Equipment}

At the present stage, China's detection method for fume purification equipment refers to "Technical Requirements and Testing Technical Specifications for Oil Fume Purifying Equipment in the Catering Industry (Trial)" (HJ/T 62-2001) and "Fume Lamp Production Emission Standards (Trial)" (GB 18483-2001). The detection system is shown in Figure 1.

(1) Introduction to detection methods

The standards require that the wind speed in the connecting ducts of the fume purification equipment should be less than $20 \mathrm{~m} / \mathrm{s}$, and the inlet and outlet connecting ducts should each have a straight section of 4.5 times the diameter (or equivalent diameter). Sampling ports are located 3 times downstream (or equivalent diameter) from elbows, reducers, etc. The fume-generating device should be able to meet the requirement that the variation of the average fume concentration within 10 minutes is less than $5 \%$, and the generation amount of soot can be adjusted to meet the requirements of different gas volume and concentration.

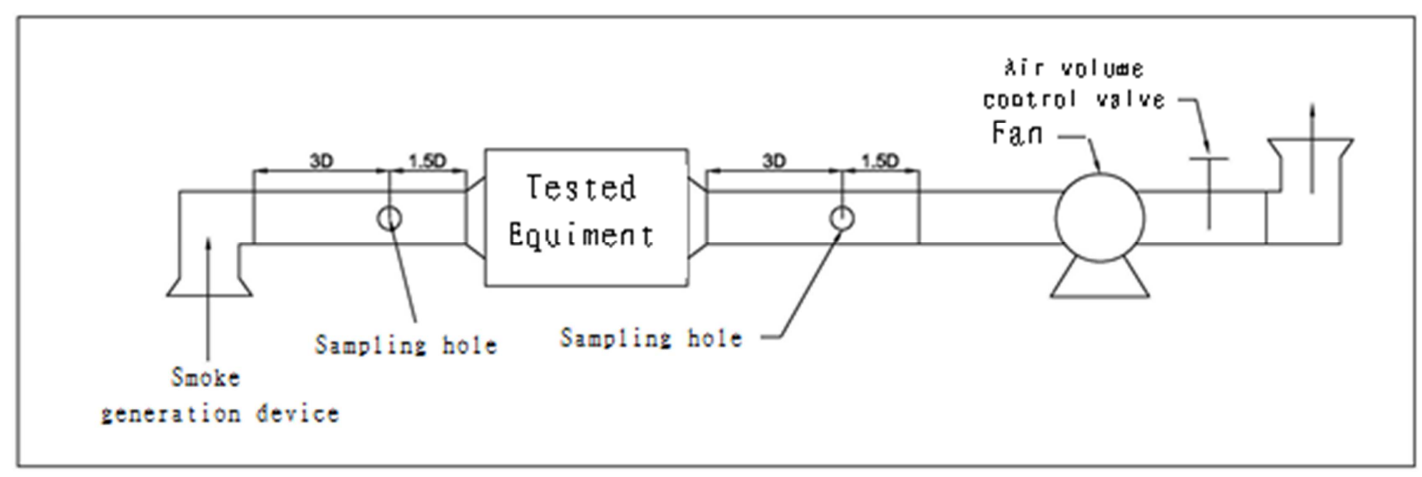

Figure 1. Fume purification equipment test system schematic.

With this test system, the use of a sampling device capable of automatically tracking the flow rate of the flue gas to achieve equal-speed sampling is used to collect the soot. The soot is collected in the filter in the collection head at a constant speed, and the soot-entrapping filter is placed on the lidded Poly 4 Within the fluoroethylene sleeve, back to the laboratory with carbon tetrachloride as a solvent for ultrasonic cleaning, moved into a quartz cell with a lid to constant volume, infrared spectrophotometric determination of soot content [2]. Calculate the volume of dry smoke in the standard state during the sampling process, and calculate the fume emission concentration after calculation.

(2) Problems that may occur during the testing process

In the soot sampling process, the air leakage rate and body resistance of the device can be obtained by comparing the flow rate of the fume purification device in and out of the air duct with the total pressure of the gas. The standard requires that the air leakage rate of the fume purification equipment body should be less than $5 \%$, the resistance of the wet type, static type equipment body should not exceed $300 \mathrm{~Pa}$, and the mechanical type and compound type should not exceed $600 \mathrm{~Pa}$. In terms of real-time monitoring of fume concentration, the existing detection devices are vulnerable to other gases, resulting in low data accuracy and poor reproducibility, affecting the accuracy of discriminating the purification efficiency of the tested devices, and the comparability of the detection results is poor, and can not become a regulatory agency on the catering industry fumes Effective technical support for emissions regulation. 


\section{Oil Smoke Purification Equipment Detection Method Optimization Ecommendations}

With the further promotion of "zero emission" and "ultra-low emission" requirements, the detection and analysis methods required in the standard can basically meet the detection and analysis of the existing purification equipment removal rate, bulk resistance, air leakage rate and other performance indicators. However, there is room for further optimization from the aspects of detection system, sampling, sampling and analysis, etc., to the purpose of precise measurement.

(1) Optimize the sampling conditions, refine the sampling process, and ensure the sampling stability of the measured samples.

In the actual operation process, the current sampling equipment in the market has poor real-time synchronization effect of isochronous sampling, and it is required to measure the laminar flow of soot in the air duct of the system. However, under operating conditions, the airflow length is difficult to achieve laminar flow in a three-fold diameter duct, especially in the air duct at the inlet end of the equipment. Because of the difference in the effect of the wind hood, it is easy to form a rotating turbulence, which affects the sampling equipment. The isokinetic acquisition. It is recommended to refer to the provisions of the "Sampling Method for Fixed Pollutant Exhaust Gases and Sampling Methods for Gaseous Pollutants" (GB/T 16157-1996). The sampling port shall be set at 6 times diameter at the downstream of the bend or reducer. It is possible to reduce the adverse effects of changes in airflow and achieve a higher constant rate of synchronous recurrence.

(2) Optimize the extract

When using infrared spectrophotometry analysis in the laboratory, the national standard requires the use of carbon tetrachloride as the extraction solution. During the analysis process, the liquid must be pipetted to the colorimetric tube several times. The carbon tetrachloride is volatile and toxic, and the liquid is removed during the process. It is extremely prone to loss and has a serious impact on the accuracy of the experimental data. In practice, reagents that are substitutes for carbon tetrachloride include hexachlorotetra fluorobutane (S-316), tetrachloroethylene, and the like. S-316 has low toxicity, high extraction rate, and is not easy to volatilize. It is an ideal extractant, but the production process is complicated and expensive. Although tetrachloroethylene is relatively cheaper than S-316, it still has high toxicity; The replacement of carbon tetrachloride by solvents that are volatile and non-toxic is an imperative for improving laboratory analysis accuracy. It is recommended that qualified units use chlorotetra fluorobutane (S-316) as the extract.

(3) Take full account of the effect of the tightness of the test duct on the air leakage rate.

In the detection of the air leakage rate, the sealing of the air duct itself was not taken into consideration. Various purification equipments were of different sizes and shapes. There were purification devices directly installed on the stoves and purification equipment installed on the outside air channels. Therefore, the connection between the air duct and the equipment to be tested in the test system becomes a problem in laboratory testing systems. It is recommended to use sealing materials such as tapering, tape, etc., and then test after confirming the sealing.

(4) The test system should increase the secondary purification device to ensure that the measured gas is completely purified and discharged.

In the entire test system specified in the standard, if the effect of the tested purification equipment fails to meet the standard, the concentration of smoke after the treatment exceeds the standard required emission limit of $2.0 \mathrm{mg} / \mathrm{m}^{3}$. Therefore, it should be configured in the laboratory detection system. The soot processing device is provided to avoid contamination of the laboratory or the external environment during the experiment and to ensure the health and safety of the experimenter.

(5) The applicability of real-time monitoring instruments for fume concentration needs to be improved.

Currently, there are light scattering methods, $\beta$-rays, and electrochemical methods for the rapid detection of particulate matter and gaseous pollutants. These methods have relatively high requirements for humidity and working conditions, and there are both particulate matter and oil droplets in oil fumes. There are gaseous smokes and the components are complex. It is a solid, liquid, and gas tri-state mixture. Even if particle and gas flue gas are detected at the same time, due to the unknown weight value of pollutants in oil fume, etc., it is still impossible to carry out on-line analysis of oil content. Therefore, the precision of real-time monitoring instruments for smoke concentration is still open to question. All of the above problems pose obstacles for the detection of soot purification equipment and supervision of soot emissions, improve the accuracy of inspection methods, and ensure the reproducibility and comparability of inspections, so that comprehensive and comprehensive evaluation of the quality of fume purification equipment can be quickly made. To achieve the purpose of effective supervision of cooking fumes.

\section{Conclusion}

The public is increasingly concerned about the fume pollution in the catering industry, and has increased the importance attached to the research and development and use of fume purification equipment. The efficient and scientific detection method is an important guarantee for testing performance indicators of purification equipment and is an important basis for formulating scientific emission standards. Through the analysis of the existing detection methods for the fumes in the catering industry, there are optimization improvements from sampling, testing, and laboratory analysis In other words, how to make the flue gas flow reach the steady, laminar flow state as much as possible when sampling, search for highly efficient and non-toxic extraction liquids, and 
improve the accuracy of particle particle fast detection methods, etc., to further improve monitoring data on the fume pollution in the catering industry Accuracy and reproducibility provide accurate data for the analysis of the quality and cleansing effects of cooking fume purification equipment. In order to develop new and efficient purification methods to meet the "zero emissions" and "ultra-low emissions" monitoring requirements, provide scientific and rigorous technical support.

\section{References}

[1] State Environmental Protection Administration. Technical requirements and testing technical specifications for cooking fume purification equipment (for trial) (HJ/T 62-2001) [S]. China Environmental Science Press, 2000

[2] State Environmental Protection Administration, State Administration of Quality Supervision, Inspection and Quarantine. Standard for fume emission in the catering industry (for trial implementation) (GB 18483-2001) [S]. China Environmental Science Press, 2000

[3] Fu Aimin, Wang Yunfei, Jia Linlin. Analysis and Study on Purification Technology of Oil Fume Waste Gas in Food Industry [J]. Environmental Science and Management, 2016, 41(09):105-108
[4] Chen Zecheng, Chen Yujun. Quality Control of Cooking Oil Purifying Equipment Certification Testing in Catering Industry [J]. China Environmental Protection Industry, 2016(06):52-54

[5] Xiang Dong, Yang Xia, Zhu Xianling, Jiang Zhanxiang, Xu Lei, Yang Qing. Experimental study on purification performance of a new type of wet impinging flow fume purification device $[\mathrm{J}]$. Energy and Energy Conservation, 2018(03): 63-65

[6] Fang Xiaoyan, Li Minghui. Some thoughts on the fume monitoring in the catering industry [J]. Resource Conservation and Environmental Protection, 2016(04):131

[7] Niu Yanlong. Research on purification technology of fume pollution in catering industry $[\mathrm{J}]$. Science and Technology Outlook, 2015, 25(29):137

[8] Zhang Yajuan. Analysis of Environmental Impact Assessment of Catering Industry Projects during Operation Period [J]. Science and Technology Innovation and Application, 2015(01):88

[9] State Environmental Protection Administration. Determination of Particles in Fixed Pollution Source Exhaust and Sampling Method of Gaseous Pollutants (GB/T16157-1996). China Environmental Science Press, 1996

[10] Chen Guofeng, Wang Wei, Zhang Huaiwei. Discussion on Application Points of "Standard for Cooking Oil Fume Emission (Trial)" (GB18483-2001) [J]. Instrumentation and Analysis Monitoring, 2016(01):44-46 